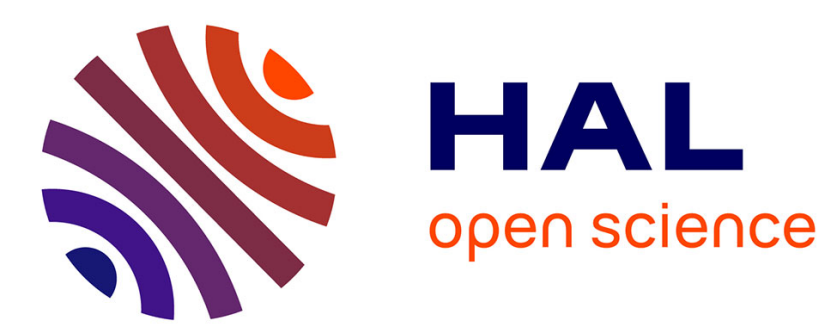

\title{
Slope-Based Event-Driven Feature Extraction For Cardiac Arrhythmia Classification
}

Julien Duforest, Benoît Larras, Deepu John, Olev Märtens, Antoine Frappé

\section{To cite this version:}

Julien Duforest, Benoît Larras, Deepu John, Olev Märtens, Antoine Frappé. Slope-Based EventDriven Feature Extraction For Cardiac Arrhythmia Classification. 2021 IEEE Biomedical Circuits and Systems Conference (BioCAS), Oct 2021, Berlin, Germany. pp.01-04, 10.1109/BioCAS49922.2021.9644957 . hal-03544633

\section{HAL Id: hal-03544633 \\ https://hal.archives-ouvertes.fr/hal-03544633}

Submitted on 26 Jan 2022

HAL is a multi-disciplinary open access archive for the deposit and dissemination of scientific research documents, whether they are published or not. The documents may come from teaching and research institutions in France or abroad, or from public or private research centers.
L'archive ouverte pluridisciplinaire HAL, est destinée au dépôt et à la diffusion de documents scientifiques de niveau recherche, publiés ou non, émanant des établissements d'enseignement et de recherche français ou étrangers, des laboratoires publics ou privés. 


\title{
Slope-Based Event-Driven Feature Extraction For Cardiac Arrhythmia Classification
}

\author{
Julien Duforest ${ }^{1}$, Benoît Larras ${ }^{1}$, Deepu John², Olev Märtens ${ }^{3}$ and Antoine Frappé ${ }^{1}$ \\ ${ }^{1}$ Univ. Lille, CNRS, Centrale Lille, Junia, Univ. Polytechnique Hauts-de-France, UMR 8520 - IEMN, Lille, France \\ ${ }^{2}$ University College Dublin, Dublin, Ireland \\ 3 'Tallinn University of Technology Institute of electronics, Tallinn, Estonia \\ e-mail : julien.duforest@junia.com
}

\begin{abstract}
To detect cardiovascular diseases (CVD), electrocardiogram (ECG) of a patient must be recorded and analyzed for a long period. For an effective diagnosis, the ECG recording system must automatically adapt to new patients. This paper presents a low-complexity artificial neural network that exclusively uses the consecutive slopes of ECG signal as inputs. These features are extracted using a level-crossing ADC and a simple TDC-based event-driven processing chain. The proposed clockless system can detect arrhythmias in ECG with 98.4\% accuracy and reduce the ANN hardware complexity by more than half compared to recent literature. It is perfectly adapted to integrated wearable monitoring systems and shows good adaptability to new patients.
\end{abstract}

Keywords-Artificial neural network (ANN), electrocardiogram (ECG), cardiac arrhythmia classification $(C A C)$, event-driven, time-to-digital converter (TDC), levelcrossing $A D C$

\section{INTRODUCTION}

Cardiovascular diseases (CVD) are one of the major causes of deaths in the world [1]. Electrocardiogram (ECG) is used for early CVD diagnosis; and wearable at-home monitoring systems can avoid patient examinations at the hospital. To enable wearable health monitoring systems with a long battery life, their power consumption must be greatly reduced. The near-sensor computing paradigm suggests that processing data in the sensor reduces the total amount of data to send to a data center for further analysis. It enables the early detection of abnormality using low-power feature extraction and classification subsystems.

Besides, ECG signals do not hold uniformly distributed information in time. Event-driven sampling, which relies on generating a sample, when a certain amplitude level is crossed, is best suited for signals such as ECG signals that have large changes in short periods and small changes over longer periods, compared to Nyquist sampling. It is demonstrated in [2] that level-crossing ADCs (LC-ADCs) offer two main advantages, compared to traditional uniform sampling, for low-to-medium resolution applications $(<8$ bits). Fewer samples are generated, which leads to less energy-hungry processing, and the majority of these samples are located at timings of interest, i.e. around the R-peaks, which are more relevant to process.

Solutions exist in the literature to classify ECG signals using non-uniform sampling. In [3], an event-driven wake-up circuit detects abnormal signals that do not fit a learned pattern, but the classification is not robust and results in an accuracy below 75\%. Moreover, no information is given on the data used for training and validation. In [4], low- complexity 96-bit features are extracted and the classification is performed with a patient-specific Artificial Neural Network (ANN). Although the reported accuracy is higher than $99 \%$, the architecture requires a global timer to calculate the precise time between two R-peaks of the ECG signal and digitally encode it as the main feature. This increases the power consumption of the system and counteracts the clockless nature of the event-driven system.

In this work, we want to combine the higher accuracy and adaptability of ANNs for classification with the simplicity and adaptability in the event-driven feature extraction stage. To that end, the slopes between two consecutive non-uniform samples are used as input features for the ANN, since the slopes hold information of both time and amplitude between samples. We demonstrate in this paper that the event-driven extraction of slope is extremely simple and that the simulated classification results in the case of premature ventricular contraction (PVC) promise accuracies up to $98.3 \%$.

This article is organized as follows. In Section II, the global architecture of the slope-based event-driven system is presented and the slope extraction, quantization, and heartbeat classification blocks are detailed. The results of arrhythmia classification is presented in Section III. Conclusions are finally drawn on the efficiency of the method.

\section{SLOPE-BASED EVENT-DRIVEN FEATURE EXTRACTION}

The input ECG signal used for testing and evaluation is extracted from the MIT-BIH arrhythmia database. The records from this database is sampled at $360 \mathrm{~Hz}$. The database is oversampled to $10 \mathrm{kHz}$ to increase the time resolution between two non-uniform consecutive samples. Figure 1 depicts an overview of the different functions implemented to perform the classification. First, it is composed of a 7-bit LC-ADC to perform the non-uniform sampling of the ECG signal. Then, a 7-state Time-to-Digital Converter (TDC) extracts the quantized values of the slopes. The framing and R-peak detection blocks select a sequence of $\mathrm{N}$ consecutive slopes centered around the R-peak of the heartbeat, before feeding them in a feed-forward fully connected ANN. It outputs either a "normal beat" or "abnormal beat" class.

\section{A. Non-uniform sampling}

The input data is non-uniformly sampled with an LCADC acting as an asynchronous delta encoder [5]. The LC- 


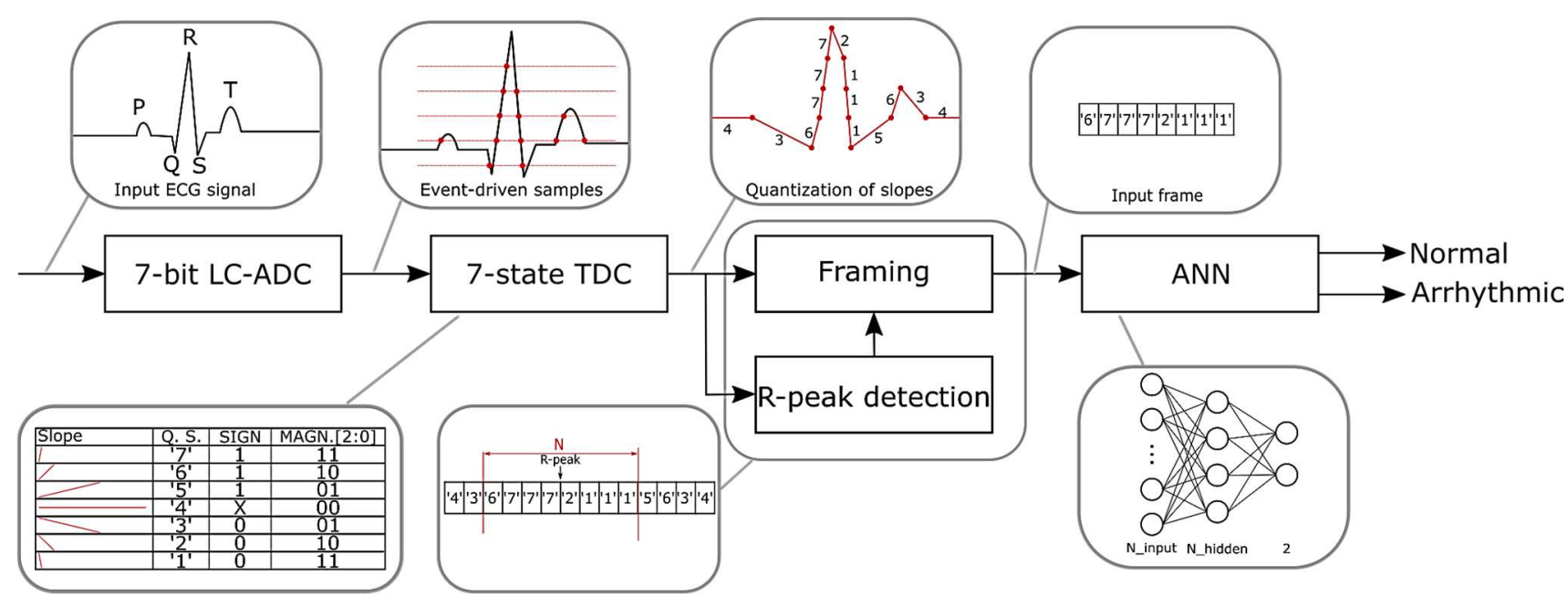

Fig. 1. Proposed event-driven architecture for the feature extraction and classification of ECG signals

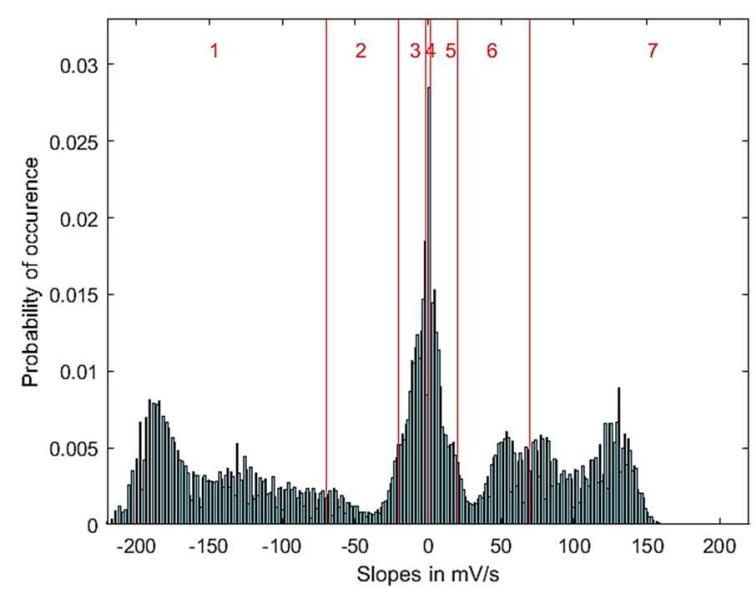

Fig. 2. Slope distribution of record 119. The vertical lines represent the slope thresholds chosen for the quantization in the TDC.

ADC outputs two signals $R E Q$ and $D I R$. REQ triggers a pulse each time a level is crossed, $D I R$ indicates the direction of the crossed level. Using a 7-bit LC-ADC, the resulting number of samples is lower than in the uniform sampling case, with an average compression ratio of 3.2 for the database records with PVCs. The LC-ADC uses a floating window, avoiding the generation of samples created by the noise around an amplitude level and guaranteeing a minimal slope between two consecutive samples.

\section{B. Time-to-digital conversion}

The non-uniform samples are converted to slopes that are quantized using a TDC. For each record, the slopes between consecutive event-driven samples are calculated. These slopes are approximated from the oversampled data as we cannot have the exact duration between two samples of the MIT-BIH database. As an example, the distribution of all the calculated slopes for record 119 is plotted as a histogram in Fig. 2. The quantization thresholds are set according to the vertical lines shown in this Figure. They divide interesting and meaningful portions of the histogram (for positive slopes) and are symmetrical around 0 to be able to use the same hardware for positive and negative slopes. For record 119, thresholds are set to $-100 \mathrm{mV} / \mathrm{s},-30 \mathrm{mV} / \mathrm{s},-4 \mathrm{mV} / \mathrm{S}, 4 \mathrm{mV} / \mathrm{s}$, $30 \mathrm{mV} / \mathrm{S}$, and $100 \mathrm{mV} / \mathrm{s}$ for a total of 7 quantized slope
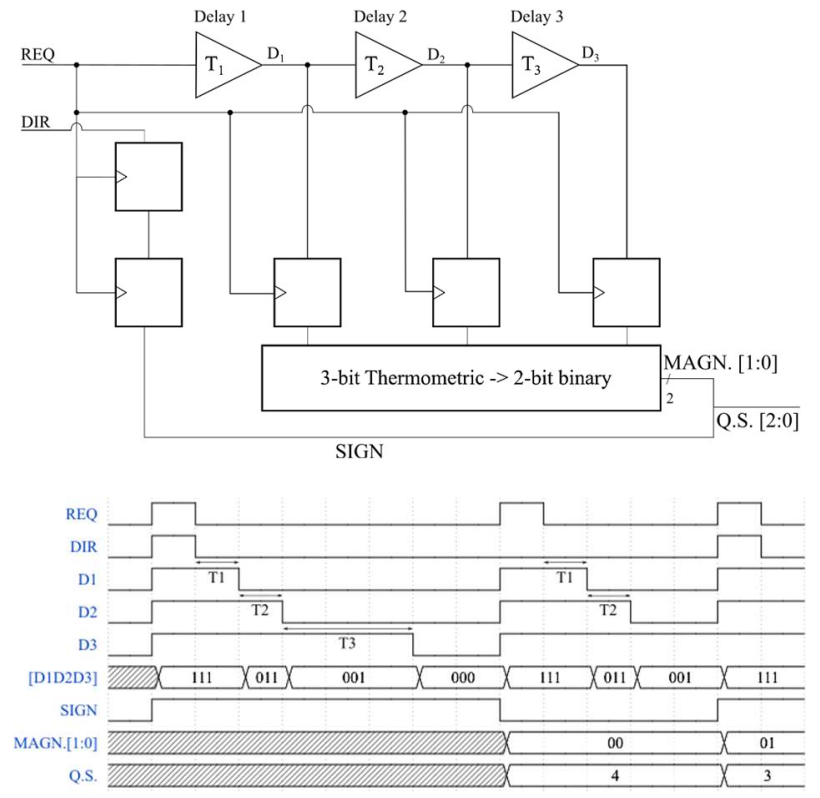

Fig. 3. Architecture and Chronogram of the TDC

values. Each distribution of slopes is patient-specific, but the histogram can always be divided as illustrated with record 119, providing 7 quantized slopes (Q.S.). In the architecture, the slope thresholds will be defined with respective delays used in the 7-state TDC. The 7 states can then be digitally coded with 3 bits, 1 for the sign of the slope and 2 for the magnitude $(M A G N$.), as shown in Fig. 1.

The architecture of the TDC is presented in Fig. 3. The block converts the REQ and DIR signals into a 3-bit nonuniformly sampled data stream at the output. The time interval between two samples, following the REQ signal, is quantized using 3 delays, corresponding to the thresholds defined previously, giving four possible quantized time ranges: $\mathrm{t}<\mathrm{T}_{1}, \mathrm{~T}_{1}<\mathrm{t}<\mathrm{T}_{2}, \mathrm{~T}_{2}<\mathrm{t}<\mathrm{T}_{3}$, and $\mathrm{T}_{3}<\mathrm{t}$.

The integrated digital delays are usually realized with thyristor-like architectures, controlled either by discharge currents [6] or by digitally-controlled capacitor arrays [7]. The delays should be configurable to adapt the slope quantization thresholds for each patient. 
The time interval between two rising edges of the REQ signal is thermometrically coded on 3 bits (D1, D2, and D3 in Fig. 3) and converted to a 2-bit binary word to constitute the magnitude $(M A G N$.) bits of $Q . S$. ., '11' for the highest magnitude and ' 00 ' for the lowest. The $S I G N$ bit is directly derived from the $D I R$ signal of the LC-ADC, 1 for a positive slope and 0 for a negative one. A second flip-flop is inserted in the SIGN path for synchronization.

It is to note that for slowly-varying signals, when $T_{3}<t$, the direction of the change signal is not relevant, thus not considered and the resulting states ('000' and '100') are aggregated into a unique state ('000').

\section{Data framing}

The data fed to the ANN is a vector of N consecutive 3bit slopes. The framing block is thus implemented by a 3-bit shift register of length $\mathrm{N}$. The center of the frame is positioned around the R-peak position, as indicated in Fig. 1, and is provided by an R-peak detection block. As a reference, R-peak detection can be performed by simple QRS detection blocks as presented in [8] or [9] with more than $97 \%$ detection rate, or by detecting the maximum of the signal reconstituted by the extracted slopes. It is to note that this latter method would be ultra-low-power but would give a reduced accuracy. In the scope of this paper, ideally placed R-peaks are considered for further classification.

\section{Heartbeat classification}

The ANN is a feed-forward fully connected neural network with an input layer with $N \_$input $=8$ neurons, a hidden layer with $N$ hidden $=4$ neurons, and two output classes (normal or arrhythmic), as shown in Fig. 1. These dimensions have been found optimal considering the tradeoff between the accuracy of the classification and the

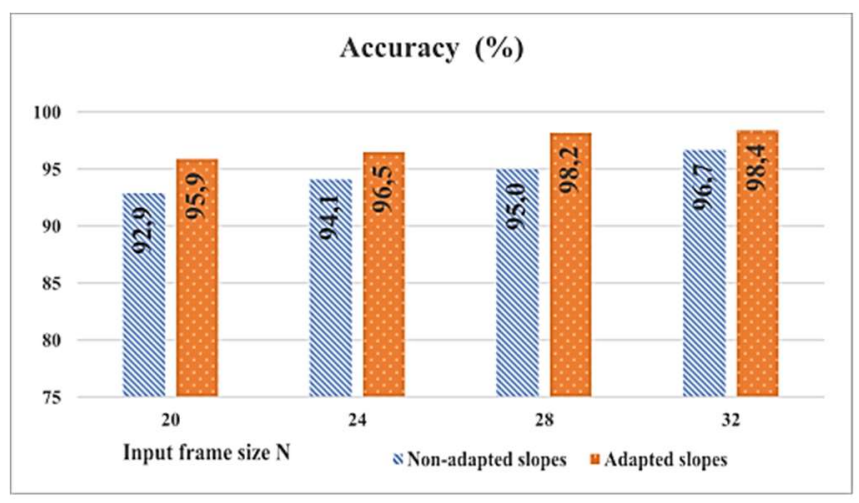

Specificity (\%)

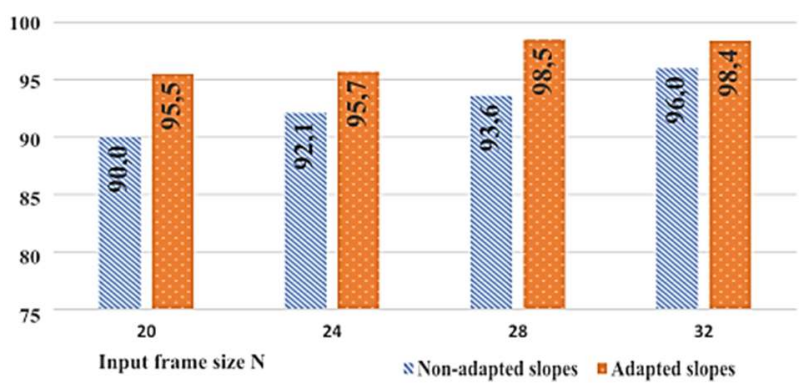

complexity of the network. The activation functions for each layer are symmetric linear functions with saturation. The output layer uses a soft maximum. Since the normal and arrhythmic classes from the ECG records are imbalanced, a conditional grouping scheme like [4] is used. Once balanced, $70 \%$ of the data is used for training, $15 \%$ for validating, and $15 \%$ for testing. The ANN is trained for each specific record independently.

\section{SIMULATION RESULTS}

\section{A. Arrhythmia classification}

The system is modeled using Matlab software and includes all the described blocks for feature extraction and classification. The records chosen for the tests are 105, 106, $109,114,119,203,205,210,215,219,221,228$, and 233 from MIT-BIH since their arrhythmias mostly consist of PVCs. The accuracy portrays how well the beats are globally classified, while the sensitivity and specificity depicts how well the normal beats and arrhythmic beats are classified, respectively. Finally, the false positive rate represents the percentage of arrhythmic beats classified as normal beats.

The classification results are given in Fig. 4 for two configurations. In the case indicated in blue, the slope thresholds are set at the values defined in Section II-B and shown in Fig. 2 for all the records. In the case indicated in orange, the slope thresholds are adapted to each record. For the different values of input frame size $\mathrm{N}$, the accuracy, sensitivity, specificity, and false positive rate are shown. In all cases, using adapted slopes leads to better classification results, but it is interesting to highlight that, even with nonadapted thresholds, the obtained accuracies are good enough to provide a low-cost embedded solution.

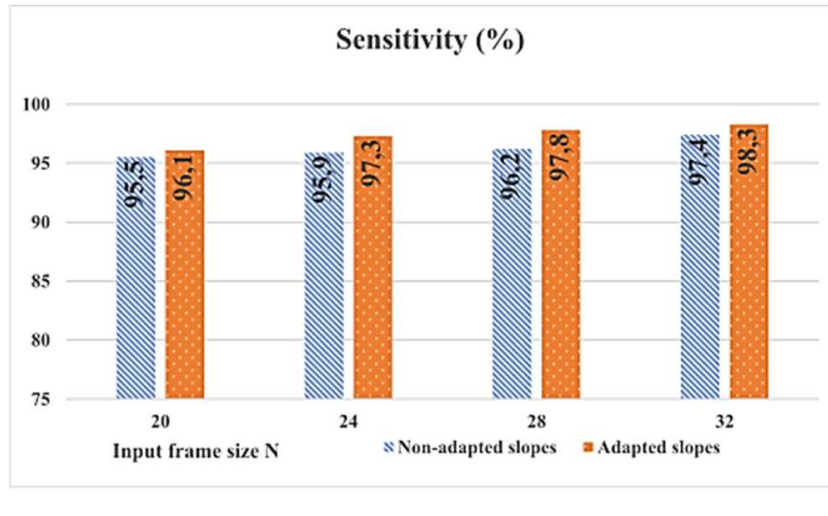

False positive rate $(\%)$

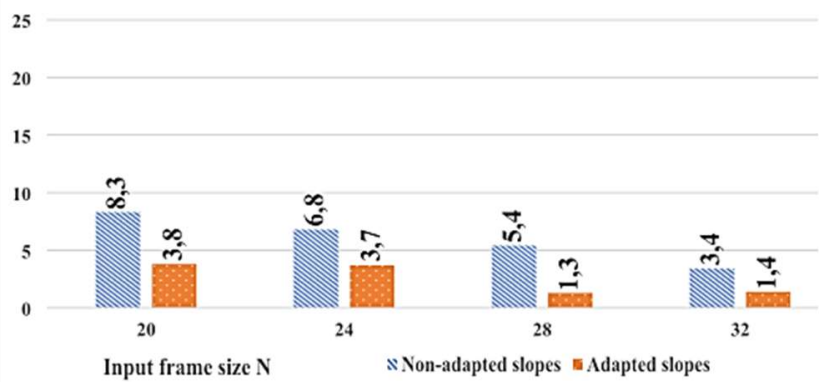

Fig. 4. Average Accuracy, Sensitivity, Specificity and False Positive Rate for all the records tested, in function of the size of the ANN input frame. In dashed blue, the slope thresholds are the same for all records. In doted orange, the slope thresholds are adapted for each record. 
TABLE I. COMPARISON OF EVENT-DRIVEN PVCS CLASSIFICATION SYSTEM

\begin{tabular}{|c|c|c|c|}
\hline & [4] & [3] & This work \\
\hline $\begin{array}{c}\text { Classification } \\
\text { Accuracy }\end{array}$ & $99.86 \%$ & $74.8 \%$ & $98.4 \%$ \\
\hline $\begin{array}{c}\text { Classification } \\
\text { operation }\end{array}$ & $\begin{array}{c}\text { ANN with 3,072 } \\
\text { accumulation and 592 } \\
\text { MAC }\end{array}$ & $\begin{array}{c}\text { 16-state finite } \\
\text { state machine }\end{array}$ & $\begin{array}{c}\text { ANN with } \\
296 \text { MAC }\end{array}$ \\
\hline $\begin{array}{c}\text { Use of RR } \\
\text { interval }\end{array}$ & Yes, coded on 11 bits & No & No \\
\hline
\end{tabular}

The maximum obtained accuracy for PVC classification is $98.4 \%$. The measured accuracies increase with the size of the frame N. For frames larger than 32, the accuracy does not improve because of the possible overlap between consecutive heartbeats. This proves that the information contained in the quantized slopes is sufficient for the correct determination of $\mathrm{PVC}$ and there is no necessity to use other information from the neighboring beats. This is an advantage for the system as the slope thresholds represent values that can be memorized on-chip, so no information is needed on top of the samples created from the LC-ADC. The threshold adaptation can increase the classification results, provided that enough samples are recorded to plot a patient-specific histogram like the one in Fig. 2. The accuracy could be increased further with a more complex selection method for the threshold or by adapting them actively during the process, but it would increase the system complexity. The ANN used in this work implements a total of $296 \mathrm{MAC}$ operations for the maximum value of the input frame size, i.e. 32 slopes.

\section{B. Comparison with state-of-the-art event-driven PVC classifiers}

As shown in Table I, the PVC classification in [3] is performed using a small 16-state finite state machine using the DIR signal from the LC-ADC along with counters and comparators. Globally, the implementation is simpler in computation compared to an ANN. However, the accuracy is 25 percentage points lower compared to other state-of-the-art classifiers and has not been tested on a cited database.

Although the work in [4] displays the best performance in terms of classification accuracy, the feature extraction operation uses an RR-interval parameter which needs to refer to the neighboring heartbeats. It can be problematic when the neighboring heartbeats are not normal beats and the classification results may be biased. Besides, to extract this parameter, a global timer and an 11-bit TDC are implemented, which is in contradiction with the event-driven character of the system.

The work presented here can reach an accuracy similar to the system in [4] (only 1.5\% lower accuracy) for the classification of PVC arrhythmias. It is done without the use of information from neighboring beats, nor the use of a global timer. The ANN computation requirements are reduced by $50 \%$ in terms of MAC operations compared to [4], which also implements 3072 additional accumulation operations.

\section{CONCLUSION}

In this paper, a classification method for arrhythmia detection is proposed specifically for wearable health monitoring. The low-complexity artificial neural network takes as input a vector of consecutive slopes extracted from the ECG signal. An efficient event-driven feature extraction architecture is derived and shows promising classification results, with up to $98.4 \%$ accuracy when the quantization thresholds are adapted to the patient. This accuracy result is very close to the state of the art, but the system offers a lower complexity, involving a classifier with less than 300 MAC operations and not requiring any information from the RR interval or neighboring beats. This work provides a promising architecture for ultra-low-power event-driven integrated health monitoring systems.

\section{ACKNOWLEDGMENT}

This work was supported by the EU Regional Development Fund (Estonian Centre of Excellence in ICT Research EXCITE TAR16013), the CHIST-ERA grant JEDAI, Mobilitas + project Mobera20, Irish Research Council, and French Research Agency project ANR-19CHR3-0005-01.

\section{REFERENCES}

[1] "Cardiovascular diseases (CVDs)." https://www.who.int/newsroom/fact-sheets/detail/cardiovascular-diseases-(cvds) (accessed Apr. 22, 2021).

[2] J. Van Assche and G. Gielen, "Power Efficiency Comparison of EventDriven and Fixed-Rate Signal Conversion and Compression for Biomedical Applications," IEEE Trans. Biomed. Circuits Syst., vol. 14, no. 4, pp. 746-756, Aug. 2020, doi: 10.1109/TBCAS.2020.3009027.

[3] G. Rovere, S. Fateh, and L. Benini, "A 2.2- $\mu$ W Cognitive Always-On Wake-Up Circuit for Event-Driven Duty-Cycling of IoT Sensor Nodes," IEEE J. Emerg. Sel. Top. Circuits Syst., vol. 8, no. 3, pp. 543554, Sep. 2018, doi: 10.1109/JETCAS.2018.2828505.

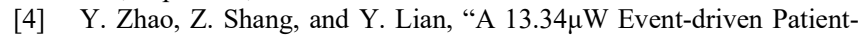
specific ANN Cardiac Arrhythmia Classifier for Wearable ECG Sensors," IEEE Trans. Biomed. Circuits Syst., pp. 1-1, 2019, doi: 10.1109/TBCAS.2019.2954479.

[5] T. Marisa et al., "Pseudo Asynchronous Level Crossing adc for ecg Signal Acquisition," IEEE Trans. Biomed. Circuits Syst., vol. 11, no. 2, pp. 267-278, Apr. 2017, doi: 10.1109/TBCAS.2016.2619858.

[6] A. González, A. Frappé, B. Larras, A. Kaiser, and P. Cathelin, "A Wide Tuning Range Delay Element for Event-Driven Processing of Low-Frequency Signals in 28-nm FD-SOI CMOS," IEEE Solid-State Circuits Lett., vol. 3, pp. 198-201, 2020, doi: 10.1109/LSSC.2020.3010877.

[7] Z. Wang et al., "20.2 A 57nW Software-Defined Always-On WakeUp Chip for IoT Devices with Asynchronous Pipelined Event-Driven Architecture and Time-Shielding Level-Crossing ADC," in 2020 IEEE International Solid-State Circuits Conference - (ISSCC), Feb. 2020, pp. 314-316. doi: 10.1109/ISSCC19947.2020.9062952.

[8] N. Ravanshad, H. Rezaee-Dehsorkh, R. Lotfi, and Y. Lian, "A LevelCrossing Based QRS-Detection Algorithm for Wearable ECG Sensors," IEEE J. Biomed. Health Inform., vol. 18, no. 1, pp. 183-192, Jan. 2014, doi: 10.1109/JBHI.2013.2274809.

[9] X. Zhang and Y. Lian, "A 300-mV 220-nW Event-Driven ADC With Real-Time QRS Detection for Wearable ECG Sensors," IEEE Trans. Biomed. Circuits Syst., vol. 8, no. 6, pp. 834-843, Dec. 2014, doi: 10.1109/TBCAS.2013.2296942. 